Portland State University

PDXScholar

3-1-2017

\title{
Some Remarks on Interpolation and Best Approximation
}

\author{
Randolph E. Bank \\ University of California, San Diego \\ Jeffrey S. Ovall \\ Portland State University, jovall@pdx.edu
}

Follow this and additional works at: https://pdxscholar.library.pdx.edu/mth_fac

Part of the Mathematics Commons

\section{Let us know how access to this document benefits you.}

\section{Citation Details}

Bank, R. E., \& Ovall, J. S. (2017). Some remarks on interpolation and best approximation. Numerische Mathematik, 1-14.

This Post-Print is brought to you for free and open access. It has been accepted for inclusion in Mathematics and Statistics Faculty Publications and Presentations by an authorized administrator of PDXScholar. Please contact us if we can make this document more accessible: pdxscholar@pdx.edu. 


\title{
Some Remarks on Interpolation and Best Approximation
}

\author{
Randolph E. Bank · Jeffrey S. Ovall
}

\begin{abstract}
Sufficient conditions are provided for establishing equivalence between best approximation error and projection/interpolation error in finite-dimensional vector spaces for general (semi)norms. The results are applied to several standard finite element spaces, modes of interpolation and (semi)norms, and a numerical study of the dependence on polynomial degree of constants appearing in our estimates is provided.
\end{abstract}

Keywords Interpolation, projection, best approximation, finite elements

Mathematics Subject Classification (2010) 65 N30, 65N15, 65N50

\section{Introduction}

It is a well established technique in finite element analysis to use interpolation error to bound the error of finite element approximations to the solutions of partial differential equations. Such scenarios also arise in other contexts, for example $L_{2}$ and other projection schemes. Let $W=\left\{w \in C(\bar{\Omega}) \cap \mathcal{H}: w_{\left.\right|_{T}} \in W(T) \forall T \in \mathcal{T}\right\} \subset \mathcal{H}$ denote a finite element space and $\mathcal{H}$ an appropriate Sobolev space. Here $\mathcal{T}$ is a partition of the domain $\Omega$ into simplicial or tensorial cells $T$, and $W(T)$ is a polynomial space defined on $T$. Let $u_{h} \in W$ be a finite element approximation to $u \in \mathcal{H}$ that satisfies the a priori error estimate

$$
\left\|u-u_{h}\right\| \leq C \inf _{\chi \in W}\|u-\chi\|
$$

for an appropriately chosen norm. Let $P: \mathcal{H} \rightarrow W$ denote an interpolation operator, usually local, typically with error estimates that are relatively easy to compute. In [3], Bank and

Bank: The work of this author was supported by the National Science Foundation under contract DMS1318480.

Ovall: The work of this author was supported by the National Science Foundation under contract DMS1414365 .

Bank: Department of Mathematics, University of California, San Diego, La Jolla, California 92093-0112. Email:rbank@ucsd.edu

Ovall: Department of Mathematics and Statistics, Portland State University, Portland Oregon, 97201. Email:jovall@pdx.edu 
Yserentant were able to show for a variety of finite element spaces and a variety of norms that

$$
\begin{gathered}
\|u-P u\| \leq C \inf _{\chi \in W}\|u-\chi\|, \\
\|u-P u\|_{T} \leq C \inf _{\chi \in W}\|u-\chi\|_{T},
\end{gathered}
$$

where $\|\cdot\|_{T}$ denotes the given norm restricted to a single element $T$ in the finite element space. Using (1)-(3), we see that

$$
C_{1}\|u-P u\| \leq\left\|u-u_{h}\right\| \leq C_{2}\|u-P u\|
$$

One may also deduce from such results that the global best approximation error is equivalent to a sum of local best approximation errors,

$$
C_{1} \inf _{\chi \in W}\|u-\chi\|^{2} \leq \sum_{T \in \mathcal{T}} \inf _{\chi \in W(T)}\|u-\chi\|_{T}^{2} \leq \inf _{\chi \in W}\|u-\chi\|^{2}
$$

as was also done by Veeser [11] in the case of the $H^{1}$ semi-norm for Lagrange finite elements by different techniques-see also [10] for a similar analysis in an energy norm associated with singularly-perturbed reaction-diffusion problems. It is the lower bound that is noteworthy. In the context of linking local interpolation with local or global best approximation, we also mention the work of Demkowicz [6], who considered various types of projection-based local interpolation schemes for several standard finite element spaces.

In this work we generalize the results (2)-(3) to include a wider class of finite elements spaces and modes of interpolation, in particular those based on integral moments as well as simple pointwise interpolation. Additionally, we provide some numerical calculations of the stability constant $\theta$ (defined below) associated with our lower bound estimates for standard families of simplicial finite elements in one and two space dimensions. Here we consider the usual nodal interpolation at both uniform and Chebyshev nodes, and a moment-based interpolation. While these calculations are restricted to the reference element, they illustrate how the stability constant $\theta$ depends on the polynomial degree $m$ of the finite element space for the important cases $\|\cdot\|_{0}$ and $|\cdot|_{1}$. We also briefly explore extensions to interpolation in vector fields for the cases of Raviart-Thomas and Nedelec spaces. Here a technical difficulty prevents the direct application of our Theorem 1 for the semi-norms $|\cdot|_{\text {div }}$ and $|\cdot|_{\text {curl }}$, but the overall conclusions of that theorem are proven to hold anyway. A numerical study of the stability constant $\theta$ with respect to $\|\cdot\|_{0}$ for Raviart-Thomas spaces in $2 \mathrm{D}$ is also provided.

\section{Main Result}

Lemma 1 Let $V$ be a finite dimensional vector space, and let $|\cdot|_{j}, j=1,2$, be two seminorms on it. We define the subspaces $N_{j}=\left\{v \in V:|v|_{j}=0\right\}$. If $N_{2} \subset N_{1}$, then there is a constant $\theta>0$ such that $|v|_{1} \leq \theta|v|_{2}$ for all $v \in V$.

Proof We consider the quotient space $V / N_{1}$, where $[v]=\left\{v+w: w \in N_{1}\right\} \in V / N_{1}$ for $v \in V$. On $V / N_{1}$ we define the norms

$$
\|[v]\|_{j}=\min _{w \in N_{1}}|v-w|_{j} .
$$


It is clear that $\|[v]\|_{j} \leq|v|_{j}$, but in fact $\|[v]\|_{1}=|v|_{1}$ because $|v-w|_{1} \geq \|\left. v\right|_{1}-\left.|w|_{1}|=| v\right|_{1}$ for $w \in N_{1}$. Finally, using the equivalence of norms on $V / N_{1}$,

$$
|v|_{1}=\|[v]\|_{1} \leq \theta\|[v]\|_{2} \leq \theta|v|_{2}
$$

which completes the proof.

Theorem 1 Let $\mathcal{H}$ be a vector space with seminorm $|\cdot|_{\mathcal{H}}$, and let $W \subset V \subset \mathcal{H}$ be finite dimensional subspaces, with

$$
N \doteq\left\{v \in \mathcal{H}:|v|_{\mathcal{H}}=0\right\} \subset W .
$$

Let $P: \mathcal{H} \rightarrow W$ and $Q: \mathcal{H} \rightarrow V$ be linear operators such that

1. $P v=v$ for all $v \in W$, and

2. $P Q v=P v$ for all $v \in \mathcal{H}$.

There is a constant $\theta \geq 1$ such that, for any $u \in \mathcal{H}$ we have

$$
|u-P u|_{\mathcal{H}} \leq \theta|u-Q u|_{\mathcal{H}}+(1+\theta) \inf _{\chi \in W}|u-\chi|_{\mathcal{H}}
$$

Furthermore, if $\theta|u-Q u|_{\mathcal{H}} \leq \beta|u-P u|_{\mathcal{H}}$ for some $\beta=\beta(u) \in[0,1)$, then

$$
|u-P u|_{\mathcal{H}} \leq \frac{1+\theta}{1-\beta} \inf _{\chi \in W}|u-\chi|_{\mathcal{H}} .
$$

Proof Because $P=I$ on $W$ and $W \supset N$, we have $N \subset\left\{v \in \mathcal{H}:|P v|_{\mathcal{H}}=0\right\}$. So the seminorms $v \mapsto|v|_{\mathcal{H}}$ and $v \mapsto|P v|_{\mathcal{H}}$ satisfy the conditions of Lemma 1 on $V$, and we have the (restricted) stability result,

$$
|P v|_{\mathcal{H}} \leq \theta|v|_{\mathcal{H}} \text { for all } v \in V
$$

for some constant $\theta>0$. For $u \in \mathcal{H}$ and $\chi \in W$, we combine (6) and the fact that $Q u-\chi \in V$ to obtain

$$
|P u-\chi|_{\mathcal{H}}=|P(Q u-\chi)|_{\mathcal{H}} \leq \theta|Q u-\chi|_{\mathcal{H}} .
$$

Therefore, it follows that

$$
\begin{aligned}
|u-P u|_{\mathcal{H}} & \leq|u-\chi|_{\mathcal{H}}+|P u-\chi|_{\mathcal{H}} \leq|u-\chi|_{\mathcal{H}}+\theta|Q u-\chi|_{\mathcal{H}} \\
& \leq(1+\theta)|u-\chi|_{\mathcal{H}}+\theta|u-Q u|_{\mathcal{H}},
\end{aligned}
$$

and (5) is a direct consequence of the additional assumption.

Remark 1 The restricted stability result (6) and the "saturation assumption",

$$
\theta|u-Q u|_{\mathcal{H}} \leq \beta|u-P u|_{\mathcal{H}} \text { for some } \beta=\beta(u) \in[0,1),
$$

are the essential ingredients of the bound (5); the containment $N \subset W$ is merely a convenient, and typical, condition that guarantees (6). We note that, if the stability result (6) were unrestricted in the sense that it held for all $v \in \mathcal{H}$, instead of just all $v \in V$, we would have the bound

$$
|u-P u|_{\mathcal{H}} \leq(1+\theta) \inf _{\chi \in W}|u-\chi|_{\mathcal{H}}
$$


immediately, without any need to make a saturation assumption. Theorem 1 replaces this much stronger stability requirement, which does not hold for many operators of interest (certainly not those considered in Section 3), with a milder stability requirement, at the cost of a saturation assumption that depends on the function $u$ under consideration. Some justification of saturation assumptions in the context of finite element computations can be found in $[5,7]$, for example.

We decompose $V$ as a direct sum in two ways,

$$
V=R \oplus N \quad, \quad V=W \oplus Z \text { where } Z=\{v \in V: P v=0\} .
$$

We note that $R$ is not uniquely determined in the first decomposition (unless $N=\{0\}$ ), but that does not affect the discussion below. We see that the optimal stability constant $\theta$ in (6) may be expressed as

$$
\theta=\max _{v_{1} \in W, v_{2} \in Z} \frac{\left|v_{1}\right|_{\mathcal{H}}}{\left|v_{1}+v_{2}\right|_{\mathcal{H}}}=\max _{v_{1} \in W \cap R, v_{2} \in Z} \frac{\left|v_{1}\right|_{\mathcal{H}}}{\left|v_{1}+v_{2}\right|_{\mathcal{H}}}
$$

One often considers the case where the seminorm $|\cdot|_{\mathcal{H}}$ is induced by a semi-innerproduct $(\cdot, \cdot)_{\mathcal{H}},|v|_{\mathcal{H}}^{2}=(v, v)_{\mathcal{H}}$. In this case, the optimal stability constant $\theta$ in (6) may be determined from the largest eigenvalue of a generalized eigenvalue problem. Given bases $W \cap R=\operatorname{span}\left\{\psi_{1}, \ldots, \psi_{m}\right\}$ and $Z=\operatorname{span}\left\{\phi_{1}, \ldots, \phi_{M}\right\}$, we have

$$
\theta^{2}=\max _{\mathbf{x} \in \mathbb{R}^{m+M}} \frac{\mathbf{x}^{T} M_{1} \mathbf{x}}{\mathbf{x}^{T} M_{2} \mathbf{x}} \quad, \quad M_{1}=\left(\begin{array}{cc}
A & 0 \\
0 & 0
\end{array}\right) \quad, \quad M_{2}=\left(\begin{array}{cc}
A & C \\
C^{T} & B
\end{array}\right),
$$

where the matrices $A, B$ and $C$ are given by

$$
a_{i j}=\left(\psi_{j}, \psi_{i}\right)_{\mathcal{H}} \quad, \quad b_{i j}=\left(\phi_{j}, \phi_{i}\right)_{\mathcal{H}} \quad, \quad c_{i j}=\left(\phi_{j}, \psi_{i}\right)_{\mathcal{H}} .
$$

The generalized eigenvalue problem (10) may be reduced in size by using the Schur complement $S=A-C B^{-1} C^{T}$,

$$
\theta^{2}=\max _{\mathbf{x} \in \mathbb{R}^{m}} \frac{\mathbf{x}^{T} A \mathbf{x}}{\mathbf{x}^{T} S \mathbf{x}}
$$

Remark 2 In all examples provided in Section 3, the matrices $A$ and $S$ are computed exactly (typically as matrices with rational entries) using Mathematica [12]; and the eigenvalue problems are solved, approximately but with high accuracy, in that environment.

Remark 3 In the context of the discussion above, suppose that a strong Cauchy inequality holds between $W$ and $Z$ for $(\cdot, \cdot)_{\mathcal{H}}$, i.e. there is a constant $\gamma \in[0,1)$ for which

$$
\left(v_{1}, v_{2}\right)_{\mathcal{H}} \leq \gamma\left|v_{1}\right|_{\mathcal{H}}\left|v_{2}\right|_{\mathcal{H}} \text { for all } v_{1} \in W \text { and } v_{2} \in Z \text {. }
$$

It follows that, for any $v_{1} \in W$ and $v_{2} \in Z,-2\left(v_{1}, v_{2}\right)_{\mathcal{H}} \leq \gamma^{2}\left|v_{1}\right|_{\mathcal{H}}^{2}+\left|v_{2}\right|_{\mathcal{H}}^{2}$, so we see that $\left(1-\gamma^{2}\right)\left|v_{1}\right|_{\mathcal{H}}^{2} \leq\left|v_{1}+v_{2}\right|_{\mathcal{H}}^{2}$. In other words, a strong Cauchy inequality implies the stability result (6),

$$
\left|v_{1}\right|_{\mathcal{H}} \leq \theta\left|v_{1}+v_{2}\right|_{\mathcal{H}} \text { for } \theta^{-2}=1-\gamma^{2} .
$$

We note that $(\cdot, \cdot)_{\mathcal{H}}$ is an inner-product on $R$, and $Z \subset R$. Now assume that there is a $\theta \geq 1$ for which $\left|v_{1}\right|_{\mathcal{H}} \leq \theta\left|v_{1}+v_{2}\right|_{\mathcal{H}}$ for all $v_{1} \in W$ and $v_{2} \in Z$. Given $v_{1} \in W$ and non-zero $v_{2} \in Z \cap R$, 
let $v_{z}=-\left(v_{1}, v_{2}\right)_{\mathcal{H}} v_{2} /\left|v_{2}\right|_{\mathcal{H}}^{2}$, so $-v_{z}$ is the orthogonal projection of $v_{1}$ onto $v_{2}$. It follows that

$$
\left|v_{1}\right|_{\mathcal{H}}^{2} \leq \theta^{2}\left|v_{1}+v_{z}\right|^{2}=\theta^{2}\left(\left|v_{1}\right|_{\mathcal{H}}^{2}-\frac{\left(v_{1}, v_{2}\right)_{\mathcal{H}}^{2}}{\left|v_{2}\right|_{\mathcal{H}}^{2}}\right) .
$$

From this, it is clear that

$$
\left(v_{1}, v_{2}\right)_{\mathcal{H}} \leq \gamma\left|v_{1}\right|_{\mathcal{H}}\left|v_{2}\right|_{\mathcal{H}} \text { for } \gamma^{2}=1-\theta^{-2} .
$$

We see, then, that (6) and (13) are essentially the same notion in this context. A saturation assumption related to (7) and a strong Cauchy inequality are the key ingredients in the traditional analysis of hierarchical basis error estimates for finite element discretizations (cf. [2]).

\section{Several Examples}

We indicate below how Theorem 1 might be applied in terms of schemes for interpolating sufficiently regular scalar or vector fields for a few families of finite element spaces. In this context, $V$ and $W \subset V$ will be members of the same finite element family, and $Q$ and $P$ will denote interpolation into $V$ and $W$, respectively, by a scheme that is fixed for the finite element family.

We use the following notation in most of the examples below. Let $T \subset \mathbb{R}^{d}$ be a closed simplex with vertices $\left\{z_{1}, \ldots, z_{d+1}\right\}$, and let $\mathcal{S}_{\ell}(T)$ denote the set of its subsimplices of dimension $\ell, 0 \leq \ell \leq d$. So $\mathcal{S}_{d}(T)=\{T\}, \mathcal{S}_{d-1}(T)$ is the set of "faces" of dimension $d-$ $1, \mathcal{S}_{1}(T)$ is the set of "edges" of dimension 1 , and $\mathcal{S}_{0}(T)=\left\{z_{1}, \ldots, z_{d+1}\right\}$ is the set of vertices, for example. For faces $F \in \mathcal{S}_{d-1}(T)$, we use $\mathbf{n}_{F}$ to denote the outward unit normal to that face. For edges $e \in \mathcal{S}_{1}(T)$ we use $\mathbf{t}_{e}$ to denote a unit tangent parallel to that edge. By $\mathbb{P}_{m}(S)$ we denote the polynomials of degree $\leq m$ on $S \in \mathcal{S}_{\ell}(T)$, and by $\tilde{\mathbb{P}}_{m}(S)$ we denote the corresponding homogeneous polynomials of degree $m$. In $\mathbb{R}^{3}$ we will also use the spaces $\mathbb{S}_{m}(T)=\left\{\mathbf{v} \in\left[\tilde{\mathbb{P}}_{m}(T)\right]^{3}: x \cdot \mathbf{v}=0\right\}$. In the examples below, we consider the (semi-)norms

$$
\begin{gathered}
\|v\|_{0}=\left(\int_{T}|v|^{2} d x\right)^{1 / 2}, \quad|v|_{1}=\left(\int_{T}|\nabla v|^{2} d x\right)^{1 / 2}, \\
|v|_{\operatorname{div}}=\left(\int_{T}(\nabla \cdot v)^{2} d x\right)^{1 / 2}, \quad|v|_{\text {curl }}=\left(\int_{T}|\nabla \times v|^{2} d x\right)^{1 / 2} .
\end{gathered}
$$

Here $|\cdot|$ denotes the Euclidean norm of a vector field or the absolute value of a scalar field.

3.1 Nodal Interpolation of Scalar Fields in Polynomial Spaces

Let $\mathcal{I}_{m}=\left\{\alpha=\left(\alpha_{1}, \ldots, \alpha_{d+1}\right) \in \mathbb{N}_{0}^{d+1}: \alpha_{1}+\cdots+\alpha_{d+1}=m\right\}$. The nodal interpolation $P_{m}: C(T) \rightarrow \mathbb{P}_{m}(T)$ is uniquely defined by

$$
\left(P_{m} v\right)\left(x_{\alpha}\right)=v\left(x_{\alpha}\right) \text { for all } x_{\alpha}=\frac{1}{m} \sum_{k=1}^{d+1} \alpha_{k} z_{k}, \alpha \in \mathcal{I}_{m} .
$$

This is the interpolation scheme considered in [3], for which we have $P_{m}=I$ on $\mathbb{P}_{m}(T)$, and $P_{m} P_{2 m}=P_{m}$ on $C(T)$ because $\left\{x_{\alpha}: \alpha \in \mathcal{I}_{m}\right\} \subset\left\{x_{\alpha}: \alpha \in \mathcal{I}_{2 m}\right\}$. For the $L^{2}$-norm we 
have $N=\{0\} \subset \mathbb{P}_{m}(T)$, and for the $H^{1}$-seminorm we have $N=\operatorname{span}\{1\} \subset \mathbb{P}_{m}(T)$, so the assumptions of Theorem 1 are satisfied in both cases.

To gain some intuition about how the stability constant $\theta$ in (6) may depend on $m$, we first numerically approximate $\theta^{2}$ for $\|\cdot\|_{0}$ and $|\cdot|_{1}$, and $1 \leq m \leq 12$, in the one-dimensional case. We lose no generality by taking $T=[-1,1]$. In addition to the uniformly-spaced nodes, $x_{k}^{(m)}=-1+2 k / m$, we also consider the Chebyshev nodes, $x_{k}^{(m)}=\cos (k \pi / m)$, although the Chebyshev nodes do not have a natural analogue in higher dimensions in this context. It is convenient in this case to use Lagrange bases $\mathbb{P}_{m}(T)=\operatorname{span}\left\{\ell_{k}^{(m)}: 0 \leq k \leq m\right\}$,

$$
\ell_{k}^{(m)}(x)=\prod_{j=0, j \neq k}^{m} \frac{x-x_{j}^{(m)}}{x_{k}^{(m)}-x_{j}^{(m)}} \quad, \quad 0 \leq k \leq m .
$$

A basis for $Z$ is $\left\{\ell_{k}^{(2 m)}: 0 \leq k \leq 2 m, k \neq 2 j\right\}$. The computed values of $\theta^{2}$ are given in Table 1 for both uniform and Chebyshev nodes. We recall that the $L^{\infty}$ Lebesgue constant (cf. [4, 9]) grows essentially exponentially for uniform nodes, and logarithmically for Chebyshev nodes, and the observed growth in $\theta^{2}$ is consistent with these rates for both of our norms.

We also consider the optimal stability constant under nodal interpolation in $\mathbb{R}^{2}$, using the reference triangle $T$ having vertices $(0,0),(1,0)$ and $(0,1)$, and the unformly-spaced nodes $x_{\alpha}$ described above. A convenient basis, in terms of nodal interpolation, for $\mathbb{P}_{m}(T)$ is the standard Lagrange nodal basis $\left\{L_{\alpha}: \alpha \in \mathcal{I}_{m}\right\}$, where $L_{\alpha}\left(x_{\alpha^{\prime}}\right)=\delta_{\alpha \alpha^{\prime}}$. Letting $\lambda_{1}=1-x-y$, $\lambda_{2}=x, \lambda_{3}=y$ denote the barycentric coordinates of $T$, we have

$$
L_{\alpha}=\prod_{j=1}^{3} \prod_{i=0}^{\alpha_{j}-1} \frac{|\alpha| \lambda_{j}-i}{\alpha_{j}-i}
$$

A basis for $Z=\left\{v \in P_{2 m}(T): P_{m} v=0\right\}$ is given by $\left\{L_{\beta}: \beta \in \mathcal{I}_{2 m} \backslash\left(2 \mathcal{I}_{m}\right)\right\}$. Using these bases, we compute the stability constants $\theta^{2}$ via the generalized eigenvalue problem (10) for both $\|\cdot\|_{0}$ and $|\cdot|_{1}$ in Table 2 , for $1 \leq m \leq 10$. As in the $1 \mathrm{D}$ case, the computed values of $\theta$ indicate exponential growth with respect to $m$.

\subsection{Interpolation of Scalar Fields in Polynomial Spaces by Integral Moments}

For interpolation by moments, we define $P_{m}: C(T) \rightarrow \mathbb{P}_{m}(T)$ by

$$
\int_{S}\left(P_{m} v\right) \kappa=\int_{S} v \kappa \text { for all } \kappa \in \mathbb{P}_{m-\ell-1}(S) \text { and all } S \in \mathcal{S}_{\ell}(T), 0 \leq \ell \leq d,
$$

where $\int_{S} v \kappa$ with $S \in S_{0}(T)$ is understood to be evaluation of $v$ at the vertex $S$. It is shown in [1], for example, that this interpolation is well-defined, with $P_{m}=I$ on $\mathbb{P}_{m}(T)$. We also see that, for any $M>m, P_{m} P_{M}=P_{m}$ on $C(T)$; because, for each $S \in \mathcal{S}_{\ell}(T), 0 \leq \ell \leq d$,

$$
\int_{S}\left(P_{m}\left(P_{M} v\right)\right) \kappa=\int_{S}\left(P_{M} v\right) \kappa=\int_{S} v \kappa=\int_{S}\left(P_{m} v\right) \kappa \text { for all } \kappa \in \mathbb{P}_{m-\ell-1}(S) .
$$

Therefore, as in the case of nodal interpolation, conditions 1)-2) of Theorem 1 are satisfied, and $N \subset \mathbb{P}_{m}(T)$ for $m \geq 0$ for standard (semi-)norms such as $\|\cdot\|_{0}$ and $|\cdot|_{1}$.

This type of interpolation is more natural than nodal interpolation in the sense that $P_{m} P_{M}=P_{m}$ for any $M>m$; we do not need $M$ to be a multiple of $m$, as we did in then 
case of nodal interpolation. Also, it has natural analogues for interpolation of vector fields in Raviart-Thomas and Nedelec spaces, as discussed below.

As with the case of nodal interpolation, we first consider the stability of interpolation by moments from $\mathbb{P}_{M}(T)$ to $\mathbb{P}_{m}(T)$ for $M>m$ in $1 \mathrm{D}$, and explicitly determine how the stability constant $\theta$ depends on $m$ and $M$ for the (semi-)norms $\|\cdot\|_{0}$ and $|\cdot|_{1}$. Again, we lose no generality by taking the interval $T=[-1,1]$. Convenient basis for $P_{m}(T)$ and $Z$ are

$$
\mathbb{P}_{m}(T)=\operatorname{span}\left\{p_{k}: 0 \leq k \leq m\right\} \quad, \quad Z=\operatorname{span}\left\{p_{k}-p_{k-2}: m+1 \leq k \leq M\right\},
$$

where $p_{k}$ is the Legendre polynomial of degree $k$ with normalization $p_{k}(1)=1$. We note that the basis for $Z$ is given in terms of (scaled) integrated Legendre polynomials.

For $\|\cdot\|_{0}$ in $1 \mathrm{D}$, the eigenvalue problem (10) can be solved explicitly,

$$
\theta^{2}=\frac{(M+1)\left(M+1-(-1)^{M-m}\right)-(m-1)(m-2)}{2(2 m-1)} .
$$

We note that, for any fixed $m, \theta$ grows linearly in $M$. A pair of functions for which $\left\|v_{1}\right\|_{0}=$ $\left\|P\left(v_{1}+v_{2}\right)\right\|_{0}=\theta\left\|v_{1}+v_{2}\right\|_{0}$ is $v_{1}=p_{m-1}$ and $v_{2}=\sum_{j=m}^{M-1} b_{j}\left(p_{j+1}-p_{j-1}\right)$, where

$$
b_{j}=\frac{(M+1)\left(M+1-(-1)^{M-m}\right)-j(j+1)}{(M+1)\left(M+1-(-1)^{M-m}\right)-(m-1)(m-2)} \frac{1+(-1)^{m-j}}{2} .
$$

For $|\cdot|_{1}$ in $1 \mathrm{D}$, we determine that $\theta=1$ as follows. For arbitrary $u \in C^{1}(T)$, we have $\int_{-1}^{1}\left(u-P_{m} u\right) \kappa d x=0$ for all $\kappa \in \mathbb{P}_{m-2}(T)$, and $\left(u-P_{m} u\right)(-1)=\left(u-P_{m} u\right)(1)=0$. Therefore,

$$
\int_{-1}^{1}\left(u-P_{m} u\right)^{\prime}\left(P_{m} u\right)^{\prime} d x=\left.\left(u-P_{m} u\right)\left(P_{m} u\right)^{\prime}\right|_{-1} ^{1}-\int_{-1}^{1}\left(u-P_{m} u\right)\left(P_{m} u\right)^{\prime \prime} d x=0 .
$$

From this, we clearly see that

$$
\left|u-P_{m} u\right|_{1}=\min _{\chi \in \mathbb{P}_{m}(T)}|u-\chi|_{1} \quad, \quad\left|P_{m} u\right|_{1} \leq|u|_{1} \quad \text { for any } u \in C^{1}[-1,1] .
$$

From this it is clear that $\left|P_{m} v\right|_{1} \leq|v|_{1}$ for all $v \in \mathbb{P}_{M}(T)$.

The stability constants $\theta^{2}$ for both $\|\cdot\|_{0}$ and $|\cdot|_{1}$ are given for $P_{m}: \mathbb{P}_{2 m} \rightarrow \mathbb{P}_{m}$ in Table 1 , for comparison with their counterparts under nodal interpolation.

For the moment-based counterparts of the 2D stability results for nodal interpolation we consider the same reference triangle $T$, but begin with bases $\left\{\lambda^{\alpha}=\lambda_{1}^{\alpha_{1}} \lambda_{2}^{\alpha_{2}} \lambda_{3}^{\alpha_{3}}: \alpha \in \mathcal{I}_{m}\right\}$ for $\mathbb{P}_{m}(T)$, and $\left\{\lambda^{\beta}: \beta \in \mathcal{I}_{2 m}\right\}$ for $\mathbb{P}_{2 m}(T)$. It is well-known that

$$
\int_{T} \lambda^{\alpha} d x=\frac{\alpha ! 2 !}{(|\alpha|+2) !}|T| \quad, \quad \int_{e_{j}} \lambda^{\alpha} d s=\frac{\alpha ! 1 !}{(|\alpha|+1) !}\left|e_{j}\right| \begin{cases}1 & , \alpha_{j}=0 \\ 0 & , \alpha_{j} \neq 0\end{cases}
$$

where $|T|=1 / 2$ is the area of $T$ and $\left|e_{j}\right|$ is the length of edge $e_{j}$ (opposite vertex $z_{j}$ ), and $\alpha \in \mathcal{I}_{r}$ for any $r \geq 0$. We also have

$$
\int_{T} \nabla \lambda^{\alpha} \cdot \nabla \lambda^{\beta} d x=\frac{2 !|T|}{|\alpha+\beta| !} \sum_{i, j=1}^{3}\left(\nabla \lambda_{i} \cdot \nabla \lambda_{j}\right) \alpha_{i} \beta_{j}\left(\alpha+\beta-e_{i}-e_{j}\right) !,
$$

where $e_{i}$ is the multi-index with 1 in its $i^{\text {th }}$ position and 0 elsewhere, and $\sigma !=0$ whenever any component of $\sigma$ is negative. 
Table 1 Stability constants $\theta^{2}$ in $L^{2}$ and $H^{1}$ for 1D nodal (uniform, Chebyshev) and moment interpolation from $\mathbb{P}_{2 m}(T)$ to $\mathbb{P}_{m}(T), T=[-1,1]$.

\begin{tabular}{cccc||cccc}
\multicolumn{4}{c}{$\left\|P_{m} v\right\|_{0}^{2} \leq \theta^{2}\|v\|_{0}^{2}$} & \multicolumn{4}{c}{$\left|P_{m} v\right|_{1}^{2} \leq \theta^{2}|v|_{1}^{2}$} \\
\hline \hline$m$ & uniform & Chebyshev & moment & $m$ & uniform & Chebyshev & moment \\
\hline 1 & 6.000 & 6.000 & 6.000 & 1 & 1.000 & 1.000 & 1.000 \\
2 & 4.375 & 4.375 & 3.333 & 2 & 1.146 & 1.146 & 1.000 \\
3 & 5.477 & 4.671 & 5.400 & 3 & 1.556 & 1.176 & 1.000 \\
4 & 5.776 & 4.283 & 4.714 & 4 & 2.728 & 1.202 & 1.000 \\
5 & 7.114 & 5.026 & 6.667 & 5 & 4.702 & 1.223 & 1.000 \\
6 & 11.64 & 4.912 & 6.182 & 6 & 9.523 & 1.267 & 1.000 \\
7 & 20.15 & 5.443 & 8.077 & 7 & 20.58 & 1.312 & 1.000 \\
8 & 40.79 & 5.394 & 7.667 & 8 & 50.26 & 1.347 & 1.000 \\
9 & 93.97 & 5.813 & 9.529 & 9 & 130.2 & 1.380 & 1.000 \\
10 & 249.0 & 5.787 & 9.158 & 10 & 366.8 & 1.408 & 1.000 \\
11 & 682.2 & 6.137 & 11.00 & 11 & 1062 & 1.434 & 1.000 \\
12 & 1972 & 6.120 & 10.65 & 12 & 3237 & 1.457 & 1.000
\end{tabular}

Using (17) for ease in computation, we construct a basis for $Z$ by applying the vertex, edge and volumetric moment conditions

$$
\lambda^{\beta}\left(z_{j}\right) \quad, \quad \int_{e_{j}} \lambda^{\beta} \lambda^{\sigma} d s \text { for } \sigma \in \mathcal{I}_{m-2} \text { with } \sigma_{j}=0 \quad, \quad \int_{T} \lambda^{\beta} \lambda^{\sigma} d x \text { for } \sigma \in \mathcal{I}_{m-3}
$$

to the basis of $\mathbb{P}_{2 m}(T)$ to form a matrix $\widehat{Z}$, whose nullspace indicates how to transform the given basis of $\mathbb{P}_{2 m}(T)$ into a basis for $Z$, and how to transform the mass and stiffness matrices for the $\mathbb{P}_{m}(T)-\mathbb{P}_{2 m}(T)$ and $\mathbb{P}_{2 m}(T)-\mathbb{P}_{2 m}(T)$ interactions to their $\mathbb{P}_{m}(T)$-Z and $Z$ - $Z$ counterparts. In the case of $|\cdot|_{1}$ a basis of $\mathbb{P}_{m}(T) \cap R$ is obtained by omitting (any) one of the given basis functions for $\mathbb{P}_{m}(T)$. The optimal stability parameters for $\|\cdot\|_{0}$ and $|\cdot|_{1}$ are given alongside their counterparts for nodal interpolation in Table 2. For both norms, the computed values are consistent with linear growth of $\theta$ with respect to $m$.

Table 2 Stability constants $\theta^{2}$ in $L^{2}$ and $H^{1}$ for $2 \mathrm{D}$ nodal (uniform) and moment interpolation from $\mathbb{P}_{2 m}(T)$ to $\mathbb{P}_{m}(T)$, for reference triangle $T$.

\begin{tabular}{ccc||ccc}
\multicolumn{3}{c}{$\left\|P_{m} v\right\|_{0}^{2} \leq \theta^{2}\|v\|_{0}^{2}$} & \multicolumn{3}{c}{$\left|P_{m} v\right|_{1}^{2} \leq \theta^{2}|v|_{1}^{2}$} \\
\hline \hline$m$ & uniform & moment & $m$ & uniform & moment \\
\hline 1 & 16.000 & 16.000 & 1 & 3.0000 & 3.0000 \\
2 & 13.195 & 18.521 & 2 & 3.3333 & 3.4500 \\
3 & 14.882 & 33.424 & 3 & 3.7910 & 4.2162 \\
4 & 22.521 & 55.275 & 4 & 6.0782 & 5.0188 \\
5 & 53.278 & 79.933 & 5 & 11.300 & 5.8320 \\
6 & 143.08 & 124.44 & 6 & 23.644 & 6.6510 \\
7 & 235.67 & 162.45 & 7 & 54.719 & 7.4737 \\
8 & 609.57 & 236.06 & 8 & 136.12 & 8.2988 \\
9 & 1391.9 & 292.58 & 9 & 365.85 & 9.1259 \\
10 & 5126.2 & 401.72 & 10 & 1055.6 & 9.9541
\end{tabular}


3.3 Interpolation of Vector Fields in Raviart-Thomas Spaces

Let $R T_{m}(T)=\left[\mathbb{P}_{m-1}(T)\right]^{d} \oplus x \tilde{\mathbb{P}}_{m-1}(T)$ be the Raviart-Thomas space of order $m$ on the simplex $T \subset \mathbb{R}^{d}$, having degrees of freedom

$$
\begin{aligned}
& \int_{F} \mathbf{v} \cdot \mathbf{n}_{F} q \text { for all } q \in \mathbb{P}_{m-1}(F) \text { and all faces } F \in \mathcal{S}_{d-1}(T), \\
& \int_{T} \mathbf{v} \cdot \mathbf{q} \text { for all } q \in\left[\mathbb{P}_{m-2}(T)\right]^{d} .
\end{aligned}
$$

Given $\mathbf{v} \in[C(T)]^{d}$, we define $P_{m} \mathbf{v} \in R T_{m}(T)$ in terms of the moments (19)-(20). It is clear again that $P_{m}=I$ on $R T_{m}(T)$ and $P_{m} P_{M}=P_{m}$ on $[C(T)]^{d}$ for $M>m$.

We begin with the semi-norm $|\cdot|_{\text {div }}$, and note from the outset that $N$, which contains gradients of all harmonic functions on $T$, is not contained in $R T_{m}(T)$ (for any $m$ ), so Theorem 1 can not be applied to guarantee the existence of a stability constant $\theta$. However, we may use the natural extension of the argument given in the case of 1D scalar interpolation by moments to show that $\theta=1$ in this case. Assuming $\mathbf{v} \in\left[C^{1}(T)\right]^{d}$, for any $q \in \mathbb{P}_{m-1}(T)$,

$$
\begin{aligned}
0 & =\int_{\partial T}\left(\mathbf{v}-P_{m} \mathbf{v}\right) \cdot \mathbf{n} q=\int_{T} \nabla \cdot\left(\left(\mathbf{v}-P_{m} \mathbf{v}\right) q\right) \\
& =\int_{T}\left(\mathbf{v}-P_{m} \mathbf{v}\right) \cdot \nabla q+\int_{T} \nabla \cdot\left(\mathbf{v}-P_{m} \mathbf{v}\right) q=\int_{T} \nabla \cdot\left(\mathbf{v}-P_{m} \mathbf{v}\right) q .
\end{aligned}
$$

Recalling that $\nabla \cdot R T_{m}(T)=\mathbb{P}_{m-1}(T)$, we deduce that $\int_{T} \nabla \cdot\left(\mathbf{v}-P_{m} \mathbf{v}\right) \nabla \cdot P_{m} \mathbf{v}=0$, so

$$
\left|P_{m} \mathbf{v}\right|_{\text {div }} \leq|\mathbf{v}|_{\text {div }} \text { for all } \mathbf{v} \in\left[C^{1}(T)\right]^{3} \supset R T_{M}(T)
$$

Therefore, we have $\theta=1$ for (6) for this choice of interpolation and semi-norm. In fact, since $P_{m} \mathbf{v}$ is already the best approximation of $\mathbf{v}$ in the $H^{\text {div }}$ seminorm, there is no need to establish (5) in this case.

We now turn to stability with respect to $\|\cdot\|_{0}$. In this case, $N=\{0\} \subset R T_{m}(T)$, so we are guaranteed a finite stability constant,

$$
\|v\|_{0} \leq \theta_{m M}^{\mathrm{div}, 0}\left\|P_{m} v\right\|_{0}
$$

A convenient basis of $R T_{m}(T)$ for our computations is

$$
\left\{\left(\lambda^{\alpha}, 0\right): \alpha \in \mathcal{I}_{m-1}\right\} \bigcup\left\{\left(0, \lambda^{\alpha}\right): \alpha \in \mathcal{I}_{m-1}\right\} \bigcup\left\{\left(\lambda^{\alpha+e_{2}}, \lambda^{\alpha+e_{3}}\right): \alpha \in \widehat{\mathcal{I}}_{m-1}\right\},
$$

where $\widehat{\mathcal{I}}_{m}=\left\{\alpha \in \mathcal{I}_{m}: \alpha_{1}=0\right\}$. In Table 3 we see the optimal stability constant for $m \leq$ 10 when $M=m+1$ and $M=2 m$. In the latter case, there is modest linear growth in $m$ throughout; but in the former case, $\theta_{m M}$ decreases up to $m=5$ before exhibiting very modest linear growth. 
Table 3 Stability constants $\left(\theta_{m M}^{\text {div }, 0}\right)^{2}$ in $L^{2}$ for $2 \mathrm{D}$ Raviart-Thomas interpolation from $\mathbb{P}_{M}(T)$ to $\mathbb{P}_{m}(T)$, for $M=m+1$ and $M=2 m$ on reference triangle $T$.

\begin{tabular}{ccc}
$m$ & $M=m+1$ & $M=2 m$ \\
\hline \hline 1 & 5.6129 & 5.6129 \\
2 & 4.6222 & 6.6839 \\
3 & 4.1060 & 9.4377 \\
4 & 4.0581 & 11.166 \\
5 & 4.0249 & 14.841 \\
6 & 4.0309 & 18.596 \\
7 & 4.0509 & 21.998 \\
8 & 4.0727 & 25.950 \\
9 & 4.0940 & 29.074 \\
10 & 4.1139 & 33.423
\end{tabular}

\subsection{Interpolation of Vector Fields in Nedelec Spaces}

Let $\mathcal{R}_{m}(T)=\left[\mathbb{P}_{m-1}(T)\right]^{3} \oplus \mathbb{S}_{m}$ be the (first-kind) Nedelec space of order $m$ on the tetrahedron $T \subset \mathbb{R}^{3}$. A function $\mathbf{v} \in \mathcal{R}_{m}(T)$ is uniquely determined by the values

$$
\begin{gathered}
\int_{e} \mathbf{v} \cdot \mathbf{t}_{e} q \text { for all } q \in \mathbb{P}_{m-1}(e) \text { and all edges } e \\
\int_{F}\left(\mathbf{v} \times \mathbf{n}_{F}\right) \cdot \mathbf{q} \text { for all } \mathbf{q} \in\left[\mathbb{P}_{m-2}(F)\right]^{3} \text { and all faces } F \\
\int_{T} \mathbf{v} \cdot \mathbf{q} \text { for all } \mathbf{q} \in\left[\mathbb{P}_{m-3}(T)\right]^{3}
\end{gathered}
$$

Given $\mathbf{v} \in[C(T)]^{3}$, we define $P_{m} \mathbf{v} \in \mathcal{R}_{m}(T)$ in terms of the moments (24)-(26). It is clear again that $P_{m}=I$ on $\mathcal{R}_{m}(T)$ and $P_{m} P_{M}=P_{m}$ on $[C(T)]^{3}$ for $M>m$.

We only consider the seminorm $|\cdot|_{\text {curl }}$ in this case. Again we see that $N \not \subset \mathcal{R}_{m}(T)$ (for any $m$ ), so we are not guaranteed a stability constant $\theta$ by the considerations given in the proof of Theorem 1. However, we establish (6) by other means, and this gives us the results (4) and (5). More specifically, it immediately follows from a well-known result (cf. [8, Lemma 5.40]) that

$$
\left|P_{m} v\right|_{\text {curl }} \leq \theta_{m M}^{\text {div }, 0}|v|_{\text {curl }} \text { for all } v \in \mathcal{R}_{M}(T)
$$

and this inequality is sharp.

\subsection{Interpolation of Scalar Fields in Tensor Product Spaces}

For a final set of experiments, we revisit nodal and moment-based interpolation schemes for tensor product polynomial spaces on the unit square $K=[-1,1]^{2}$. We denote the polynomials of degree at most $m$ in each variable on $K$ by $\mathbb{Q}_{m}(K)$. As before, we provide a comparison between the stability constants for the different modes of interpolation from $\mathbb{Q}_{2 m}(K)$ to $\mathbb{Q}_{m}(K)$. The three modes of interpolation are:

1. Nodal interpolation on the uniform lattice $\{(2 i / m-1,2 j / m-1): 0 \leq i, j \leq m\}$.

2. Nodal interpolation on the Chebyshev lattice $\{\cos (i \pi / m), \cos (j \pi / m)): 0 \leq i, j \leq m\}$. 
3. Moment-based interpolation $v \mapsto w=P_{m} v$ defined by

$$
w( \pm 1, \pm 1)=v( \pm 1, \pm 1) \quad, \quad \int_{K} w \kappa d x=\int_{K} v \kappa d x \text { for all } \kappa \in \mathbb{Q}_{m-2}(K)
$$

Each of these modes of interpolation satisfy $P_{m} P_{2 m}=P_{m}$. The stability constants are given with respect to the (semi-)norms $\|\cdot\|_{0}$ and $|\cdot|_{1}$, as well as the "energy" norm $\|\cdot\|_{\varepsilon}=$ $\|\cdot\|_{0}+\varepsilon|\cdot|_{1}$ typically associated with singularly-perturbed reaction-diffusion problems. Letting $h K=[-h, h]^{2}$ and $\tilde{v}(x)=v(h x)$ for $x \in[-1,1]^{2}$ and $v \in H^{1}(h K)$, we note that

$$
\|v\|_{L^{2}(h K)}^{2}+\varepsilon\|v\|_{H^{1}(h K)}^{2}=h^{2}\|\tilde{v}\|_{L^{2}(K)}^{2}+\varepsilon\|\tilde{v}\|_{H^{1}(K)}^{2} .
$$

So if we choose $h=\sqrt{\varepsilon}$, the stability constant for the energy norm on $h K$ is the same as if we measured it on $K$ with $\varepsilon=1$. As such, we use only the domain $K$ for the energy norm results, and use $\varepsilon=1,10^{-2}, 10^{-4}$.

In the case of the $L^{2}$-norm, the tensorial nature of the spaces imparts a simple Kronecker product structure to the associated generalized eigenvalue problem (10). A consequence of this is that the $L^{2}$ stability constant for a given $m$ is precisely the square of its counterpart in the $1 \mathrm{D}$ case. This simple squaring of the $1 \mathrm{D}$ stability constant does not carry over to the $H^{1}$-seminorm, or the energy norm. In Table 4 we have the squares of the optimal stability constants with respect to $L^{2}$ and $H^{1}$ for each of the three modes of interpolation, and in Table 5 we consider the energy norm but use only uniform nodal interpolation and moment interpolation. $\mathrm{n}$ the lowest-order case, $m=1$, the three modes of interpolation are equivalent, and we have

$$
\theta^{2}=\max \left\{\frac{3(2+11 \varepsilon)}{1+18 \varepsilon}, \frac{18\left(2+40 \varepsilon+25 \varepsilon^{2}\right)}{1+45 \varepsilon+450 \varepsilon^{2}}\right\}
$$

for the energy norm. For $\varepsilon<1.47563$, the first term in the maximum is dominant. One observes that the limiting values $\varepsilon=0$ and $\varepsilon \rightarrow \infty$ agree with the $L^{2}$ and $H^{1}$ values, respectively.

Table 4 Stability constants $\theta^{2}$ in $L^{2}$ and $H^{1}$ for nodal and moment interpolation from $\mathbb{Q}_{2 m}(K)$ to $\mathbb{Q}_{m}(K)$, for unit square $K$.

\begin{tabular}{cccc||cccc}
\multicolumn{4}{c}{$\left\|Q_{m} v\right\|_{0}^{2} \leq \theta^{2}\|v\|_{0}^{2}$} & \multicolumn{4}{c}{$\left|Q_{m} v\right|_{1}^{2} \leq \theta^{2}|v|_{1}^{2}$} \\
\hline \hline$m$ & uniform & Chebyshev & moment & $m$ & uniform & Chebyshev & moment \\
\hline 1 & 36.000 & 36.000 & 36.000 & 1 & 1.8333 & 1.8333 & 1.8333 \\
2 & 19.140 & 19.140 & 11.111 & 2 & 1.7861 & 1.7861 & 1.9047 \\
3 & 29.993 & 21.820 & 29.160 & 3 & 2.9163 & 2.6031 & 2.2183 \\
4 & 33.367 & 18.346 & 22.224 & 4 & 8.7585 & 3.0168 & 2.6004 \\
5 & 50.608 & 25.265 & 44.444 & 5 & 23.411 & 3.4931 & 3.1032 \\
6 & 135.40 & 24.132 & 38.215 & 6 & 83.593 & 3.6965 & 3.3990 \\
7 & 406.01 & 29.630 & 65.237 & 7 & 349.23 & 4.0049 & 3.9638 \\
8 & 1663.7 & 29.091 & 58.778 & 8 & 1866.1 & 4.1406 & 4.2275 \\
9 & 8830.8 & 33.796 & 90.810 & 9 & 11580 & 4.3785 & 4.8121 \\
10 & 61996 & 33.485 & 83.867 & 10 & 86657 & 4.4838 & 5.0615
\end{tabular}


Table 5 Stability constants $\theta^{2}$ in energy norm, $\left\|Q_{m} v\right\|_{\varepsilon}^{2} \leq \theta^{2}\|v\|_{\varepsilon}^{2}$, with $\varepsilon=1,10^{-2}, 10^{-4}$ for nodal and moment interpolation from $\mathbb{Q}_{2 m}(K)$ to $\mathbb{Q}_{m}(K)$, for unit square $K$.

\begin{tabular}{ccc||ccc||ccc}
\multicolumn{4}{c}{$\varepsilon=1$} & \multicolumn{4}{c}{$\varepsilon=10^{-2}$} & \multicolumn{3}{c}{$\varepsilon=10^{-4}$} \\
\hline \hline$m$ & uniform & moment & $m$ & uniform & moment & $m$ & uniform & moment \\
\hline 1 & 2.4314 & 2.4314 & 1 & 28.926 & 28.926 & 1 & 35.910 & 35.910 \\
2 & 1.7917 & 1.9620 & 2 & 8.4409 & 7.1232 & 2 & 18.864 & 11.041 \\
3 & 2.9315 & 2.2557 & 3 & 7.4385 & 6.5525 & 3 & 28.485 & 27.727 \\
4 & 8.7927 & 2.6149 & 4 & 12.027 & 4.3714 & 4 & 31.105 & 20.491 \\
5 & 23.441 & 3.1171 & 5 & 26.501 & 4.4560 & 5 & 47.633 & 34.524 \\
6 & 83.580 & 3.4051 & 6 & 84.372 & 4.0549 & 6 & 121.80 & 27.390 \\
7 & 349.03 & 3.9699 & 7 & 337.35 & 4.5693 & 7 & 377.99 & 34.022 \\
8 & 1864.6 & 4.2303 & 8 & 1763.3 & 4.5526 & 8 & 1601.0 & 27.381 \\
9 & 11571 & 4.8155 & 9 & 10898 & 5.1464 & 9 & 8809.5 & 28.839 \\
10 & 86596 & 5.0634 & 10 & 81693 & 5.2518 & 10 & 61632 & 23.825
\end{tabular}

\section{References}

1. Arnold, D.N.: Spaces of finite element differential forms. In: F. Brezzi, P. Colli-Franzone, U.P. Gianazza, G. Gilardi (eds.) Analysis and Numerics of Partial Differential Equations, Springer INdAM Series, vol. 4, pp. 117-140. Springer, Milan (2013). DOI 10.1007/978-88-470-2592-9_9. URL http://dx.doi. org/10.1007/978-88-470-2592-9_9

2. Bank, R.E.: Hierarchical bases and the finite element method. In: Acta numerica, 1996, Acta Numer., vol. 5, pp. 1-43. Cambridge Univ. Press, Cambridge (1996)

3. Bank, R.E., Yserentant, H.: A note on interpolation, best approximation, and the saturation property. Numer. Math. 131(1), 199-203 (2015). DOI 10.1007/s00211-014-0687-0. URL http://dx.doi. org/10.1007/s00211-014-0687-0

4. Brutman, L.: On the Lebesgue function for polynomial interpolation. SIAM J. Numer. Anal. 15(4), pp. 694-704 (1978). URL http: //www. jstor .org/stable/2156848

5. Carstensen, C., Gallistl, D., Gedicke, J.: Justification of the saturation assumption. Numerische Mathematik 134(1), 1-25 (2016). DOI 10.1007/s00211-015-0769-7. URL http://dx.doi.org/10.1007/ s00211-015-0769-7

6. Demkowicz, L.F.: Polynomial exact sequences and projection-based interpolation with application to Maxwell equations. In: D. Boffi, L. Gastaldi (eds.) Mixed Finite Elements, Compatibility Conditions, and Applications, Lecture Notes in Mathematics, vol. 1939, pp. 101-158. Springer Berlin Heidelberg (2008). DOI 10.1007/978-3-540-78319-0_3. URL http://dx.doi.org/10.1007/978-3-540-78319-0_3

7. Dörfler, W., Nochetto, R.H.: Small data oscillation implies the saturation assumption. Numer. Math. 91(1), 1-12 (2002)

8. Monk, P.: Finite element methods for Maxwell's equations. Numerical Mathematics and Scientific Computation. Oxford University Press, New York (2003). DOI 10.1093/acprof:oso/9780198508885.001. 0001. URL http://dx.doi.org/10.1093/acprof : oso/9780198508885.001.0001

9. Smith, S.J.: Lebesgue constants in polynomial interpolation. Ann. Math. Inform. 33, 109-123 (2006)

10. Tantardini, F., Veeser, A., Verfürth, R.: Robust localization of the best error with finite elements in the reaction-diffusion norm. Constr. Approx. 42(2), 313-347 (2015). DOI 10.1007/s00365-015-9291-5. URL http://dx.doi.org/10.1007/s00365-015-9291-5

11. Veeser, A.: Approximating gradients with continuous piecewise polynomial functions. Found. Comput. Math. pp. 1-28 (2015). DOI 10.1007/s10208-015-9262-z. URL http://dx.doi.org/10.1007/ s10208-015-9262-z

12. Wolfram Research Inc.: Mathematica 10.0 (2014) 